\title{
La utilización de las TIC para la difusión de Proyectos Científicos de Género ${ }^{1}$
}

\author{
Mariló Berenguer Ros
}

marilo.berenguer@ua.es

Universidad de Alicante

Mariló López Tébar

mariadolores.lopez@ua.es

Universidad de Alicante

Recibido: 28-02-2013

Aceptado: 19-03-2013

\section{Resumen}

En nuestro proyecto "Haciendo Historia: Género y Transición en España", las fuentes deben tener una característica común: aportar datos nuevos. Para lo cual, pretendemos recuperar, reconstruir, preservar y difundir las experiencias vividas por "ellas", una representación importante de las mujeres que fueron protagonistas de ese momento histórico pero ignoradas por los medios de comunicación. El proyecto recoge testimonios mediante la realización de entrevistas a las protagonistas. Al mismo tiempo, adquirimos todos aquellos documentos que puediesen enriquecer las entrevistas, para digitalizarlos y formar parte de los datos aportados. Y, puesto que la mejor manera de fomentar el conocimiento es poniéndolo a disposición de todo el mundo, el proyecto final vendrá en formato web para su difusión vía Internet.

Palabras clave: Cms, web, medios audiovisuales, comunicación, testimonios, difusión, investigación.

\begin{abstract}
In our project "Haciendo Historia: Género y Transición en España", the sources must have a common feature: bring new information. In order to accomplish that, we try to recover, rebuild, preserve and disseminate "their" experiences, a significant representation of women that took part in this historical moment but ignored by the media. The project includes testimonies by conducting interviews with the protagonists. At the same time, we have acquired all documents that could enrich the interviews, and scan them to become part of the information provided. In addition, since the best way to promote knowledge is making it available to everyone, the final project will come in web format for its dissemination through the Internet.
\end{abstract}

Key words: Cms, web, audiovisual media, communication, testimonies, diffusion, research.

\footnotetext{
${ }^{1}$ Este estudio ha sido realizado en el marco del Proyecto de investigación 'Haciendo Historia: Género y Transición en España', fianciado por el MICINN (Ref. FEM2010-19068).
} 
La era de la información es nuestra era. Es un período histórico caracterizado por una revolución tecnológica centrada en las tecnologías digitales de información y comunicación, concomitante, pero no causante, con la emergencia de una estructura social en red, en todos los ámbitos de la actividad humana, y con la interdependencia global de dicha actividad. Es un proceso de transformación multidimensional que es a la vez incluyente y excluyente en función de los valores e intereses dominantes en cada proceso, en cada país y en cada organización social. Como todo proceso de transformación histórica, la era de la información no determina un curso único de la historia humana. Sus consecuencias, sus características dependen del poder de quienes se benefician en cada una de las múltiples opciones que se presentan a la voluntad humana (Manuel Castells, 2005).

\section{Introducción}

El proyecto "Haciendo Historia: Género y Transición en España", surge por un objetivo común e interdisciplinar que consiste en la recuperación de la experiencia vital, personal y social de las protagonistas de nuestra historia reciente: las mujeres anónimas, las mujeres sindicalistas, las mujeres en los partidos políticos, las mujeres parlamentarias, las mujeres educadoras... de la Transición española.

La recogida de testimonios de mujeres permite un enriquecimiento de la historia al completar, con una presencia femenina, el anterior vacío. Pero sobre todo hace posible replantear cuestiones y conceptos básicos como democracia, igualdad, ciudadanía, acción colectiva, militancia o identidad, que tradicionalmente se han interpretado en clave sólo masculina. De esta manera podría incluirse en los análisis históricos y políticos elementos como el acceso real, no sólo formal, la ciudadanía política y social, la presencia desigual en los puestos de poder, la asimetría en las formas de militancia en función del género, el cuestionamiento de la división rígida entre lo público y lo privado, etc.

Los testimonios audiovisuales resultan de gran importancia para el recuerdo de colectivos olvidados como el de las mujeres y para recoger experiencias, valoraciones y actitudes que no suelen aparecer en las fuentes escritas y, mucho menos todavía, en las sonoras. Las palabras de dirigentes políticas, integrantes de movimientos sociales y de mujeres anónimas tienen una presencia todavía poco destacada.

Se trata de conocer a esas mujeres a través de su imagen, de su relato, de verlas, de escuchar la historia por boca de ellas mismas. De conocer sus vidas a través de sus palabras, de sus gestos y de sus documentos y de difundirlas, de darlas a conocer, de acercarlas tanto a personas investigadoras como a navegantes anónimos, a instituciones, a fundaciones, a la sociedad.

Somos cada día más conscientes de que el avance tecnológico modifica constantemente la vida de las personas, sus códigos de comunicación y formas de socialización.

Existen cada vez más dispositivos tecnológicos que actúan modificando las formas de interacción humanas. Nuevos medios de comunicación dan lugar a nuevas maneras de 
relaciones interpersonales, nuevas tendencias, nuevas formas de almacenar y de transmitir la información y, por lo tanto, nuevas maneras de comprenderla, de entenderla...

El visionario canadiense Marshall McLuhan, a finales de los años 60, anticipó que la investigación debería orientarse hacia nuevas formas de experiencia y hacia el descubrimiento de principios de organización universalmente aplicables, una aldea global que describía una sociedad futura en la que todos estaríamos comunicados mediante las herramientas tecnológicas.

Esa sociedad futura es nuestra sociedad actual. Sociedad en la que vamos creando nuevas maneras de comunicación, creando tecnologías que van condicionando nuestro pensamiento y nuestra conducta; en la que vamos creando los contenidos y creando los medios para comunicarlos.

Estas formas nuevas de comunicación rompen las barreras de las distancias, llevando el conocimiento más allá de cualquier frontera física; no se trata sólo de transmisión sino también de producción de conocimiento. No debemos olvidar que la sociedad es el fin último del trabajo investigador y es a la sociedad a la que de forma directa o indirecta va dirigido ese trabajo.

Y aquí radica la parte más innovadora de nuestro proyecto de investigación, la utilización de las TIC (Tecnologías de la Información y la Comunicación) y de la red Internet para la difusión, transmisión y desarrollo de la investigación. Sólo en la interacción entre personas conocidas y desconocidas tiene sentido el proyecto.

En la parte técnica estamos trabajando en una primera fase, que contempla dos tipos de acciones:

1. La recogida de material.

Consiste en la grabación de las entrevistas (vídeo y audio), el escaneado de los documentos cedidos para el proyecto y el registro de toda la actividad mediante fotografías para añadir al informe.

2. Portal Web.

Creación de un portal web para la publicación y difusión del contenido de la investigación.

\section{Recogida del material}

Para la realización del primer punto, nos hemos tenido que dotar de Tecnología de Alta Definición (HD) por lo que significa (bien definida, precisa, sólida, detallada) para el registro audiovisual de los testimonios de nuestras protagonistas.

Nos desplazamos un mínimo de tres personas hasta el lugar de la entrevista con el equipo técnico formado por cámara de vídeo HD, trípode de cámara, baterías, micrófono de solapa inalámbrico, auriculares, tarjetas compact flash, foco para iluminación, trípode para 
foco, sistema de grabación backup para entrevista (Átomos Ninja), escáner, ordenador portátil y cámara de fotos.

El equipo humano está compuesto por dos técnicas, encargadas de la grabación y del registro de la documentación, y varias investigadoras que son las que realizan la entrevista.

Los pasos que seguimos en la producción y realización de la entrevista son los siguientes: localización y primer contacto con la entrevistada a la que se le pone en antecedentes del proyecto y de quienes somos.

Disponemos de una pequeña muestra de algunos testimonios recogidos en los siguientes enlaces web: http://www.mujeresytransicion.es/ y http://www.youtube.com/watch?v=gbR$2 \mathrm{VrpBCI}$.

Concretamos día y hora y nos desplazamos hasta el sitio acordado. Una vez allí, nos presentamos y le detallamos el proyecto, también nuestra intención de colocar a disposición de cualquier persona o institución, su historia particular. Solicitamos la cesión de documentos para el proyecto y una de las técnicas comienza el escaneo y datación en el ordenador portátil mientras la otra técnica y la entrevistadora, junto con la entrevistada, deciden el lugar de la entrevista.

Intentamos, siempre que nos sea posible, realizar la entrevista en su casa. Buscamos un lugar en el que se encuentren espacialmente cómodas; probamos iluminación y sonido y solamente eliminamos del entorno del encuadre de la cámara los elementos que pueden causar una distorsión en el plano.

Comenzamos la grabación de la entrevista: Pedimos que se presenten y nos cuenten sus antecedentes familiares para situarlas en un contexto familiar y social además de histórico. De esa manera, antes de comenzar el tema objeto de nuestro estudio, las entrevistadas se han acostumbrado a nuestra presencia y a nuestras preguntas.

A partir de ese momento nos centramos en el tema del proyecto, "La Transición Española". Las investigadoras, desde sus respectivas áreas de estudio, han consensuado un guión con preguntas para cada una de las entrevistadas. Eso supone realizar un estudio previo y pormenorizado de las protagonistas de nuestro proyecto de investigación.

La realización de las entrevistas nos permite establecer relaciones de amistad basadas en intereses comunes, que no terminan al finalizar la entrevista sino que van, en algunos casos, más allá del proyecto en sí.

\section{Portal Web (TebasCMS)}

Cuando se comenzó a estudiar cómo queríamos representar, transmitir y hacer visible todo el proyecto la conclusión fue que el soporte web era el idóneo para ello.

El lenguaje iba ser nuestro protagonista. El lenguaje como medio de transmisión. Pero ¿en qué formato? ¿Lenguaje escrito, lenguaje oral, lenguaje corporal, lenguaje visual...? La 
respuesta era lógica: Todos ellos con todas sus características y sus aportaciones tenían que estar presentes.

La palabra debía ser leída, oída y visualizada, sólo de esta forma podría haber un proceso de comunicación pleno, una construcción de identidades y relaciones, para despertar a la reflexión y para que al mismo tiempo se produjera ese performance entre un narrador frente a un público, es decir que la difusión de este conocimiento, de estos testimonios, debía de disponer de un amplio elenco de recursos escénicos, y combinarlos de una manera interactiva, para que finalmente quedara completamente representado todo aquello que todavía no había sido contado, o sí, pero nunca antes publicado.

Además todos estos objetivos tenían que quedar recogidos en diferentes tipos de material, los llamados multimedios: texto, imagen, sonido, vídeo en movimiento, ... étc.

Estos multimedios debían ser expuestos a través de un canal. Sólo un canal oral y un canal visual eran insuficientes. Necesitábamos un canal de comunicación que uniera las características de ambos. Este canal debía ser digital, porque a través de él, y:

"gracias al soporte técnico que proporciona la cibernética por medio de las unidades de información y comunicación numéricas binarias — bit es abreviatura de 'binary digit', es decir, dígito o número binario-, es posible llevar a cabo una comunicación que es a la vez visual y acústica y que cuenta con elementos lingüísticos escritos (elementos visuales lingüísticos), con elementos lingüísticos orales (elementos acústicos lingüísticos), con elementos visuales no lingüísticos y con elementos acústicos no lingüísticos. El canal digital es un canal multimedia, acústico y visual, lingüístico y no lingüístico, en el que se sitúa y se produce la comunicación de construcciones lingüísticas orales y escritas, así como de sonidos no lingüísticos, construcciones musicales, construcciones visuales, etc. La digitalidad es la cualidad digital de este canal" (IV Congreso de la CiberSociedad: Crisis analógica, futuro digital, 2009).

Así mismo esta digitalidad y:

"una característica que es intrínseca a ella: la computabilidad, convierten a las tecnologías actuales en tecnologías para el procesamiento de la información, cualquiera que sea la naturaleza del soporte y cualquiera que sea la morfología de dicha información. De manera que toda esta información puede ser tratada de forma rápida al ser procesada como datos computables por el ordenador" (Lamarca Lapuente, 2011).

Resumiendo: sea cual sea la morfología de la información analógica anterior (textos impresos, fotografías, vídeos, etc.), ahora puede ser convertida en bits de información, esto es, transformada en información digital para poder ser manipulada y tratada por el ordenador.

Y este último punto, es lo que nos lleva a la representación mediante este canal, mediante la web. La web como herramienta para que este proyecto sea procesado, sea material de consulta y de análisis, un material testimonial de la memoria de esas mujeres para su preservación, reconstrucción y difusión. Bajo una herramienta hipermedia que integre todos estos hipermedios, de tal modo que se entrelacen para formar un continuo de información e interacción virtualmente infinito y que además exista la posibilidad de interactuar con los usuarios. 
Analizando que tipo de sitio web se adecuaba a todos nuestros requerimientos finalmente nos decimos por un CMS. Un CMS (Content Management System), es lo que se conoce como un sistema de gestión de contenidos, que es un programa que permite crear una estructura de soporte para la creación y administración de contenidos de páginas web. Se trata de una interfaz que controla una o varias bases de datos donde se aloja el contenido del sitio web. Estos sistemas permiten tratar de manera independiente el diseño del contenido y, de esta forma, permiten que el autor no se tenga que preocupar del diseño. Es decir, podrá crear, clasificar y publicar cualquier tipo de información en el sitio web, pero no tendrá que preocuparse de cómo gestionar su visualización, de pelearse con el código fuente de la página ni con las particularidades de una programación web.

Por lo tanto, un CMS estará compuesto de dos partes, un back y un front, siendo el back la parte donde los administradores editan y publican las informaciones y el front la parte donde los visitantes visualizan las mismas.

Dentro de la cantidad de CMS disponibles en el mercado, era necesario un CMS específico, un CMS dedicado a la ingesta y gestión de contenidos audiovisuales. El elegido para tal tarea fue finalmente TebasCMS.

Figura1. Página principal portal “Mujeres y Transición” (Portal en desarrollo).

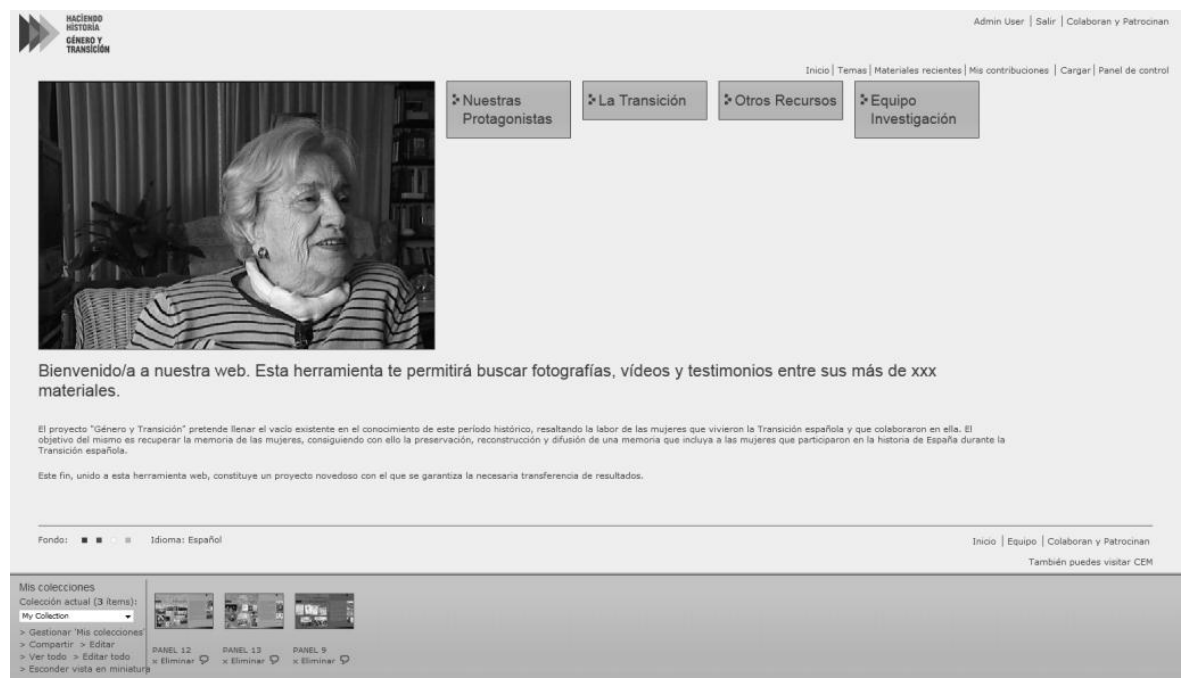

Fuente: Haciendo Historia: Género y Transición en España (2013).

\section{1. ¿Por qué ha sido necesaria una aplicación de gestión de contenidos de esta índole?}

"Cotidianamente se hace uso de gestores de contenido, ya sea cuando se escribe un artículo en un Blog, cuando se publica un video en YouTube o simplemente cuando se busca un contacto en una agenda de teléfonos. La pregunta debía ir más encaminada a qué tipo de aplicación de gestión de contenido necesitábamos. 
¿Necesitábamos organizar una colección de fotografías?, para lo cual, podría haber bastado con un "Adobe Bridge" o si nos inclinamos por aplicaciones gratuitas por "Google Picasa", no hubiesen sido ninguna mala opción, de hecho incluso nos permitía compartir colecciones de fotos en la web a través de álbumes. ¿Necesitábamos trabajar también con vídeo? YouTube es una opción muy válida para publicar los vídeos incluso poder crearnos varios canales según temática. De igual manera existen herramientas que nos permiten gestionar documentos y clips de audio, que serían casi todas las tipologías de recursos multimedia con las que seguro nos debíamos enfrentar. Pero que ocurre ¿cuando tenemos que gestionar todos esos contenidos de una forma relacionada?, ¿qué pasa cuando necesito información específica contenida digitalmente en los archivos?, ¿qué sucede cuando mi trabajo me obliga a generar colecciones de contenido con recursos de diversos tipos, relacionados entre sí y con formas de explotación y publicación diversas? TebasCMS, es un gestor heterogéneo que admite múltiples tipos de recursos bajo la misma aplicación, un interface web que me permite acceder a los contenidos desde cualquier parte del mundo, un gestor de contenidos con suficiente inteligencia para identificar el tipo de recurso con que le estoy alimentando y extraer información del mismo. Una herramienta que tiene un comportamiento distinto si trabajo con fotografía, extrayendo información de metadato (datos que describen otros datos) digital de los ficheros de imágenes, o bien extrayendo el texto y documentarlo en base de datos, si es que estoy trabajando con documentos. Una aplicación que dispone de herramientas que me permiten realizar anotaciones en base al código de tiempo de un vídeo concreto y luego me permite buscar por texto libre, y localiza el punto exacto del vídeo donde anoté esa información. Con ella es posible organizar el contenido mediante campos de documentación, creando colecciones de contenido y temas, que agrupen a su vez estas colecciones. TebasCMS tiene algunos mecanismos automáticos para crear estas agrupaciones y para introducir metadato en el sistema de forma automática.

Otro punto a tener en cuenta, seria las necesidades de cada usuario en cuanto a documentación, cada uno tendrá la necesidad de organizar el contenido de una determinada manera, en base al uso que le quiera dar a la información que este analizando, no necesitará los mismos campos de documentación aquel usuario que realice una investigación sobre un tema histórico, que aquel que haga clipping publicitario, o una aplicación de "Catch Up", así que resultaría muy difícil satisfacer las necesidades de todos los usuarios, incluso planteando un esquema de documentación dinámico y parametrizable, ya que en un interface de aplicación Windows como es el cliente Tebas, sería poco práctico acceder a la información.

Por otro lado la herramienta tiene que permitirnos gestionar usuarios, ya que si las aplicaciones del contenido están dentro de nuestra web, que queremos sea referencia para la consulta y tratamiento de material de interés, se dará el caso de que los usuarios necesiten acceder a ese contenido, y debemos disponer de herramienta que gestione usuarios, sus credenciales y nivel de acceso, así como las herramientas que puede usar y las que no, que calidades del contenido puede ver, y cuales puede descargar" (Alonso, 2011). 
Figura 2. Detalle de un recurso tipo vídeo en el portal web.

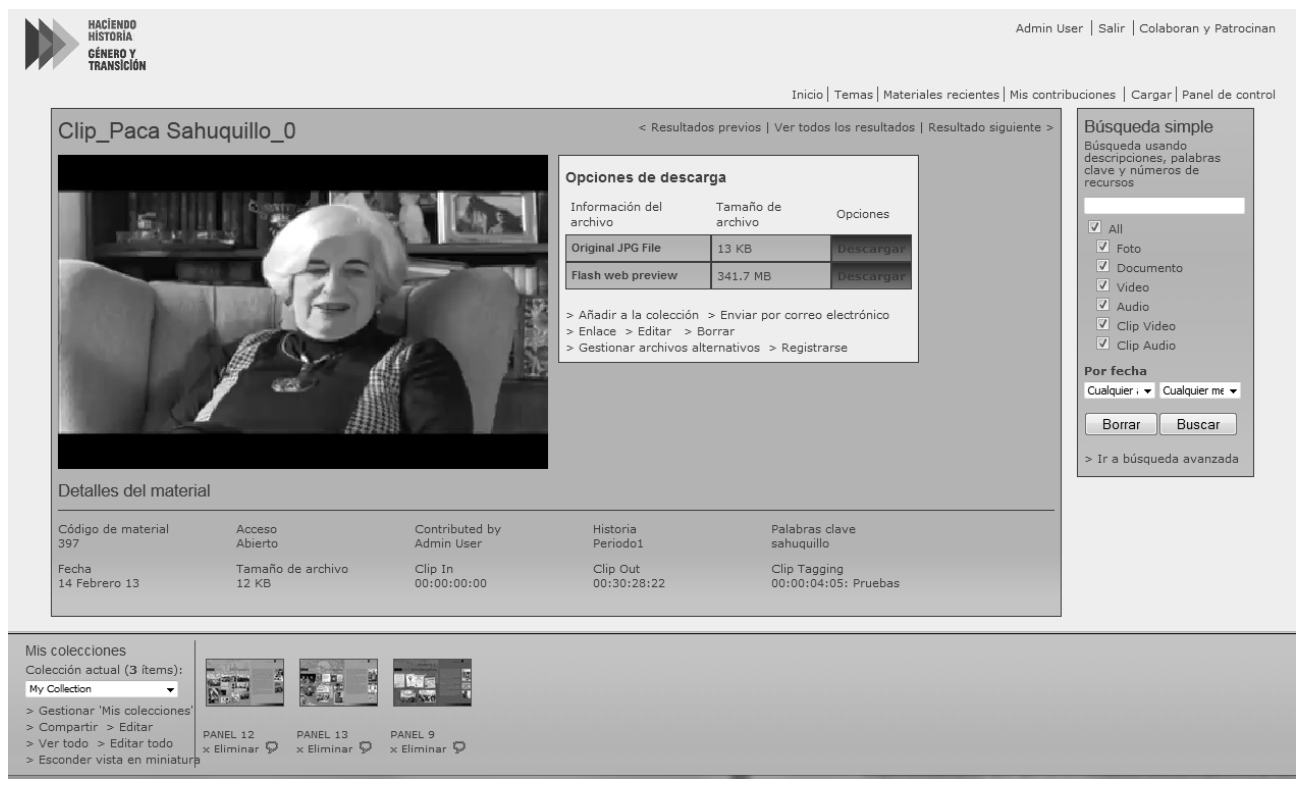

Fuente: Haciendo Historia: Género y Transición en España (2013).

\subsection{Importancia de los Metadatos para las búsquedas}

"El metadato es el centro neurálgico para la gestión de contenido dentro de la estructura fundamental de TebasCMS, existen otras formas de organizar nuestro material, como disponerlo en una estructura de carpetas o categorías, tal y como haríamos en un sistema de ficheros en nuestro PC. Otra posibilidad es organizar el contenido teniendo en cuanta el tipo de contenido que es, pero estas dos formas de organización son solo válidas hasta cierto punto, ya que permiten organizar nuestro contenido en un solo sentido; por ejemplo: organizar todos los vídeos en una categoría, las fotografías en otra, los documentos en otra y el audio en otra, o bien hacer categorías en base a proyectos y incluir dentro de ellas todos los vídeos, fotografías y documentos que pertenecen a cada proyecto. En cualquiera de estas dos formas, nos encontraremos en una situación de "rigidez organizativa" con respecto a nuestro contenido, rigidez que sufriremos a la hora de buscar, cuando se nos plantee la necesidad de obtener del sistema los vídeos relativos a un proyecto, ciertas fotografías del mismo, más algunas fotografías pertenecientes a otro proyecto y realizar una nueva agrupación con este material.

Usando la documentación (metadato) como primer nivel de organización, podemos clasificar nuestro contenido de varias formas simultáneamente, de manera que puedo disponer de contenido, por ejemplo de vídeo, que pertenezca por un lado a la categoría de "divorcio" y por otro lado tener ese mismo vídeo organizado en una colección de todas aquellas noticias que tengan relación con el de "transición" y conseguir que ese vídeo aparezca en los resultados de mis búsquedas cuando busco por cualquiera de los dos criterios. 
La diferencia fundamental entre estas dos estructuras organizativas, radica en buscar contenido o tratar de encontrarlo. Hay muchos sistemas de gestión donde el usuario dispone su contenido, organizado por sus criterios y para encontrarlo recorre un árbol de categorías que la mayoría de las veces se hace excesivamente extenso y complejo, o tiene que plantearse la pregunta de si el material que está buscando estaba disponible como vídeo, fotografía o documento. De esta manera el gestor de contenido tiene que tener una idea muy clara de cómo está organizado y qué contenido tiene, con lo que muchas de las veces se convierte en el "amo del calabozo" y resulta casi imposible encontrar algo en el sistema si no es él quien lo busca.

Un sistema de gestión de contenido debe atender las peticiones de todos los usuarios, sin la necesidad de que sepan que hay en el sistema y como encontrarlo, una búsqueda simple donde poder especificar las claves del contenido que está buscando. Si necesitamos una fotografía de una manifestación, introducimos "manifestación" en la caja de búsqueda del sistema y debe aparecernos todo el contenido etiquetado como tal. Además podremos buscar por "manifestación mujer pancarta lema" y deberemos obtener un contenido en el que aparezca una manifestación en el que aparece una mujer portando una pancarta con un determinado lema, por ejemplo.

Lo ideal para la definición de un esquema de documentación, es la definición de listas de valores, expresados en listas desplegables o selectores de etiquetas donde reflejemos información sobre origen, idioma, personajes, categorías, acciones, tipos de plano, emociones. Este método es mucho más eficiente que un campo de texto libre, donde el usuario que inserta la información puede cometer errores tipográficos, o lo que es más complicado, poner a todos los usuarios de acuerdo en el criterio de documentación libre; con términos fijos, no hay opción a cometer errores y está mucho más nítido el concepto de anotación.

Otro aspecto importante es añadir metadato al sistema "lo antes posible" incluso antes de disponer del material en sí, de manera que generamos contenido sin la necesidad de tener un archivo media asociado. Debemos añadir el metadato en el proceso de ingesta y aprovecharnos de los métodos automáticos de extracción de esa información disponibles en TebasCMS. Podemos aprovechar el propio nombre del fichero del recurso para extraer información sobre qué tipo de media es, simplemente por el tipo de archivo, pero además podemos codificar el nombre del mismo y usar una convención de datos que permita a TebasCMS catalogar el contenido desde su entrada en el sistema, incluso relacionarlo con otros contenidos.

Los métodos de extracción de información digital como etiquetas Exif (Exchangeable image file format que es una especificación para formatos de archivos de imagen usado por las cámaras digitales) y similares, permiten a TebasCMS saber muchas cosas sobre el contenido y volcar esa información sobre campos de documentación del esquema de datos. Podemos obtener información, también, de la estructura de directorios desde la cual importamos el archivo media en el sistema, asociando los nombres de las carpetas a categorías u otros campos de documentación" (Alonso, 2010a). 
Figura 3. Resultado de una búsqueda en el portal web.

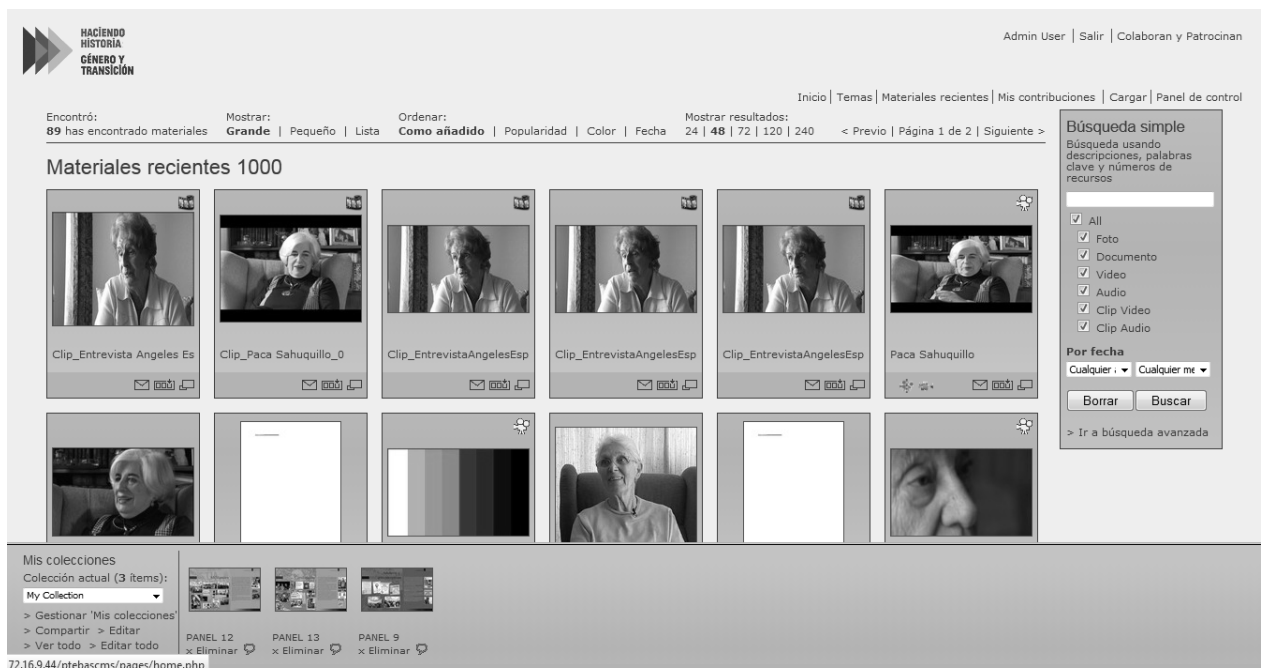

Fuente: Haciendo Historia: Género y Transición en España (2013).

\subsection{Gestión de usuarios}

"Una de las necesidades más importantes de un Administrador ya sea un administrador de sistemas, o bien un administrador de contenidos, consiste en poder definir una serie de permisos para sus usuarios, ya sean permisos a nivel de acceso o bien a nivel de funcionalidad con respecto al contenido. Estos permisos, en definitiva, van a describir el rol del usuario dentro de la plataforma y la interacción que este realizará con el sistema. Es muy importante poder agrupar usuarios según su rol o actividad y poder establecer relaciones entre estos grupos de usuarios. El administrador puede encontrarse con distintos flujos de trabajo, según el tamaño de la compañía o el uso que se requiera hacer del contenido, y es por este motivo que, una herramienta de gestión de usuarios y permisos, debe permitirle configurar el sistema para poder adaptarlo a todas y cada una de las necesidades a las que deba dar respuesta. Desde la más sencilla mediateca hasta las más compleja plataforma de gestión, explotación y distribución, con capacidades "multicliente" donde podemos organizar el contenido de diferentes contribuyentes, con la posibilidad de que los usuarios solo puedan acceder al contenido que les corresponde, y tener un nivel de acceso que permita acceder a la calidad original del contenido o bien a vistas previas del mismo.

TebasCMS dispone de una gestión de usuarios y grupos, a los que podemos asignar privilegios o permisos, jerarquizar los grupos entre sí, heredar permisos de los grupos superiores o definir comportamientos del sistema basados en esos permisos. Definir, por ejemplo usuarios que consulten los contenidos en baja calidad, y deban hacer una solicitud de los contenidos que les resultan interesantes, para que se les envíen dichos contenidos en alta calidad. Podremos definir flujos de trabajo complejos, con contribuyentes de contenido 
localizados en cualquier parte del mundo o en nuestro propio departamento de producción, adaptar el sistema para un reducido número de usuarios o bien gestionar grupos de trabajo con roles determinados, en el caso de que dividamos en equipos de trabajo las distintas tareas de ingesta, documentación, búsqueda de contenido o archivado del mismo.

Desde los contribuyentes hasta los consumidores del contenido de nuestro sistema, el Administrador debe tener un minucioso control de las posibilidades y estados, por lo que un contenido pasa a lo largo de su vida, TebasCMS dispone de una gran variedad de métodos de "carga" de contenido, ya sea de un solo contenido a través de un simple "upload" o bien mediante procesos por lotes, a través de sus componentes web, que permiten la selección de múltiples ficheros y su carga en el sistema mediante un proceso automático. Podemos recibir el contenido, mediante FTP o bien destinar una carpeta local en el servidor, para que los usuarios internos puedan dejar allí su contenido y éste sea ingestado por el sistema. Podemos establecer métodos de catalogación automática dependiendo de por qué método insertemos contenido en el sistema, o en qué nivel de nuestra carpeta de ingesta se encuentre el contenido.

Si queremos definir un flujo de trabajo complejo, podemos establecer grupos de trabajo, hacer un grupo con permisos específicos para revisar el contenido que entra en el sistema y realizar aprobaciones del mismo, haciendo así que esté disponible para todos o determinado grupo de usuarios. Podemos definir el contenido como Público o Privado y dejar que sea el usuario, que ha contribuido dicho contenido, el que decida cuando y como compartirlo. Permitir que otros usuarios hagan peticiones de contenido que no se encuentra aún en el sistema, definiendo un formulario de petición en el que se recoja la información necesaria para poder elaborar el contenido, saber en qué tiempo es necesario tener disponibilidad de él, qué tipo de contenido es necesario para ese proyecto y en qué formato se precisa.

Desde el punto de vista de un gestor o administrador de contenido, muchas veces es necesario disponer de un interface multilenguaje, debido a que tenemos consumidores de distintos países, que no solo precisan entender qué es lo que están haciendo en el sistema si no que, además, necesitan buscar contenido en su lenguaje nativo, por lo que además de un interface multilenguaje, el metadato deber serlo también. TebasCMS dispone de un gestor de contenido que permite introducir tanto los textos de la aplicación como las opciones de catalogación en múltiples lenguajes.

Es común la necesidad de definir campos de documentación dependiendo de los usuarios o la temática del contenido. TebasCMS dispone de una herramienta de configuración, de nuestra ficha de documentación, en la que podemos definir qué campos deseamos crear para cada tipo de contenido, y qué campos deseamos crear, comunes a todo tipo de contenido, qué grupos de usuarios acceden a esos campos, y qué grupo de usuarios, además de ver la documentación de esos campos, tienen permiso para editarla.

En muchos de los sistemas existentes, la metodología de modificación de datos para un grupo determinado de contenido, pasa por la necesaria edición de los campos que queramos modificar, en la ficha de cada uno de los contenidos, mientras que TebasCMS dispone de la posibilidad de editar los valores de uno o varios campos de documentación en un solo proceso 
de edición, para uno, un grupo o todos los contenidos del sistema, en una sola acción" (Alonso, 2010b).

Figura 4. Detalle de Panel de Control desde donde configura la herramienta un usuario Administrador.

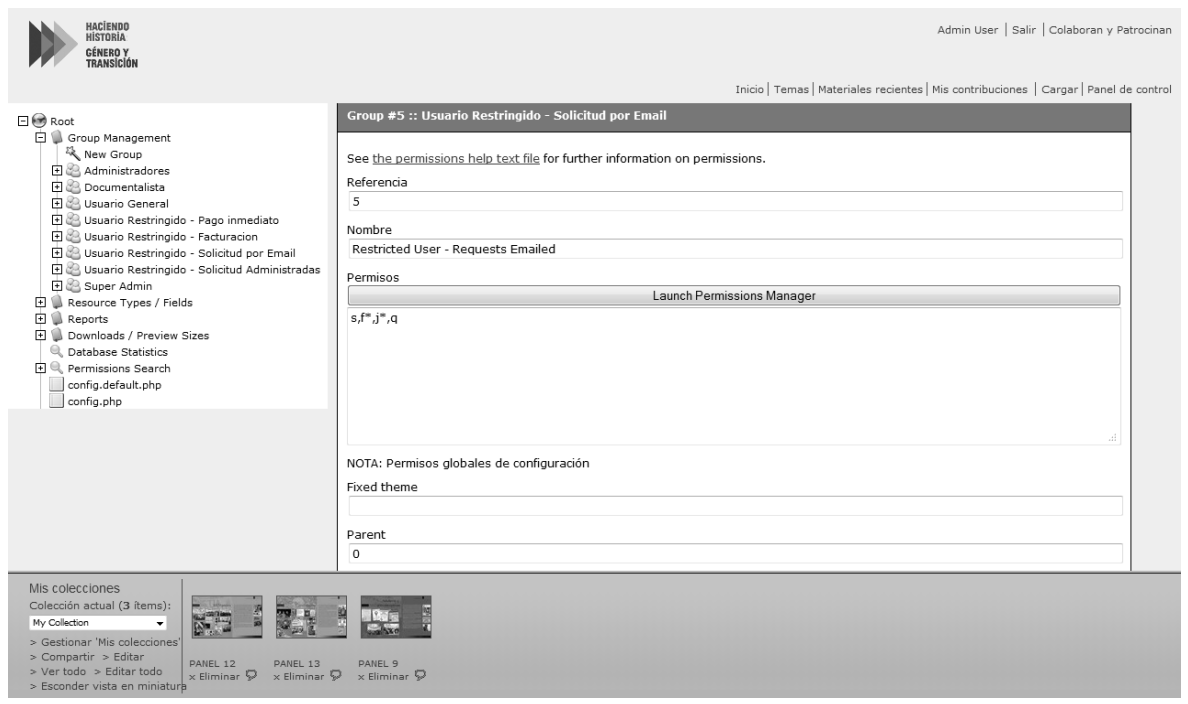

Fuente: Haciendo Historia: Género y Transición en España (2013).

\section{Conclusiones}

TebasCMS reúne una serie de características que hemos considerado importantes a la hora de elegir esta plataforma, que pretendemos que satisfaga a todos aquellos usuarios que deban trabajar con contenido audiovisual:

- "TebasCMS tiene que ser un gestor de contenidos heterogéneo, capaz de trabajar y "comprender" tanto contenido audiovisual, como contenido abstracto o paquetes de contenido.

- Debe estar basado en un interface sencillo, actual, de última tecnología y común a todos los usuarios como es un entorno web.

- Debe estar orientado a la gestión de grandes cantidades de contenido, a una gestión sencilla y eficaz del mismo y a una búsqueda rápida del mismo.

- Tiene que estar orientado al metadato como primer nivel de organización de material, con independencia del tipo de contenido del que se trate.

- Los contenidos deben ser encontrados de forma rápida y sencilla. En caso de aceptar peticiones externas de contenido, el equipo de trabajo debe disponer de las herramientas para hacerlo con eficacia y rapidez, es mejor enviar "enlaces" al 
contenido que está en el sistema, que utilizar correo electrónico o FTP, y que los usuarios se los descarguen del sistema.

- Debe gestionar los contenidos en su calidad más alta, optimizando así los procesos de trabajo y garantizando el acceso al material de mayor calidad.

- Un repositorio central, bien organizado debe ser el núcleo de nuestro archivo.

- La colaboración y uso compartido de los materiales es esencial en el trabajo del día a día.

- Un entorno de estadísticas e informes debe ayudar a comprender el uso de nuestra plataforma y controlar el contenido dentro de ella. Un entorno de trabajo intuitivo, visualmente rico y orientado a la productividad.

- Completamente Web con la única necesidad de un navegador sin tener que instalar ninguna aplicación en la estación de trabajo.

- Compatible con lo estándar, tanto a nivel de sistema operativo como de navegadores Web sin importar en que entorno trabajemos.

- Búsquedas simples y avanzadas, presentes en todo momento en el interface principal, permitiendo encontrar rápidamente los contenidos, utilizar búsquedas avanzadas para refinar los resultados o incluso búsqueda por similares para ser más efectivo.

- Inteligencia a la hora de ordenar los resultados de las búsquedas, haciéndolo por

el peso especifico de las palabras clave, los contenidos más buscados aparecen siempre en primer lugar.

- Una información detallada sobre el contenido, campos de documentación, imagen previa, icono o distintos tamaños de visualización.

- Colaboración entre usuarios, compartiendo contenido y organizando para trabajo en grupo.

- Acceso directo a todas las calidades del contenido, con posibilidad de descarga de las distintas calidades.

- Organización del contenido en Temas y Colecciones.

- Búsqueda página por página en documentos PDF, extracción e indexación de texto de documentos generados digitalmente" (Alonso, 2010c). 


\section{BIBLIOGRAFÍA}

- IV Congreso de la CiberSociedad: Crisis analógica, futuro digital (2009): "Sobre el texto literario y el canal de la comunicación: oralidad y escritura, digitalidad y multimedialidad. La edición de textos clásicos", [en línea] Disponible en: http://www.cibersociedad.net/c ongres2009/es/coms/sobre-el-texto-literario-y-el-canal-de-la-comunicacion-oralidad-yescritura-digitalidad-y-multimedialidad-la-edicion-de-textos-clasicos/891/ [18/12/2012].

- Alonso, Carlos (2010a): "La importancia del Metadata", [en línea] Disponible en: http://tebas.techex.es/?tag=busqueda [13/02/2013].

. (2010b): “TebasCMS - Beneficios para el Administrador", [en línea] Disponible en: http://tebas.techex.es/?tag=gestion-de-usuarios [20/02/2013].

. (2010c): "TebasCMS - El Gestor de Contenidos de la familia Tebas", [en línea]

Disponible en: http://tebas.techex.es/?tag=archivo-y-documentacion [13/02/2013].

. (2011): “TebasCMS: ¿Cuánto cuesta una gestión de contenidos?”, [en línea] Disponible en: http://tebas.techex.es/?tag=gestor-de-contenidos [13/02/2013].

- Castells, Manuel. (2005): Guión de presentación en el V Foro Social Mundial. Porto Alegre, 29 enero 2005, [en línea] Disponible en: http://www.cic.unb.br/ rezende/trabs/castellsVFSM.html [13/02/2013].

- Haciendo Historia: Género y Transición en España (2013): [en línea] Disponible en: http://www.mujeresytransicion.es/adelanto.html [10//02/2013].

- McLuhan, Marshall (2009): Comprender los medios de comunicación. Las extensiones del ser humano. Barcelona: Paidós.

- Lamarca Lapuente, María Jesús. (2011): "Hipertexto: el nuevo concepto de documento en la cultura de la imagen". Tesis doctoral, [en línea] Disponible en: http://www.hipertexto.info/documentos/digital.htm [11/02/2013]. 